\title{
Enteral nutrition management in stroke patients: a narrative review
}

\author{
Liya Gong, Yan Wang, Jian Shi \\ Department of Nutrition, The Second Hospital of Hebei Medical University, Shijiazhuang, China \\ Contributions: (I) Conception and design: L Gong; (II) Administrative support: Y Wang; (III) Provision of study materials or patients: L Gong, Y \\ Wang; (IV) Collection and assembly of data: All authors; (V) Data analysis and interpretation: All authors; (VI) Manuscript writing: All authors; (VII) \\ Final approval of manuscript: All authors. \\ Correspondence to: Liya Gong, MM. Department of Nutrition, The Second Hospital of Hebei Medical University, 215 Heping West Road, Xinhua \\ District, Shijiazhuang, China. Email: yoya0820@163.com.
}

\begin{abstract}
Objectives We aimed to summarize the enteral nutrition $(\mathrm{EN})$ management of stroke patients according to recent evidence.

Background: Stroke patients have a high incidence of dysphagia, which is the main cause of malnutrition, and stroke with malnutrition leads to high recurrence and mortality. Insufficient food intake caused by dysphagia is the main cause of malnutrition in stroke patients, which is associated with poor prognosis, increased mortality, and deteriorated outcomes in patients with stroke. Dehydration is also worthy of attention.

Methods: Non-systematic searches of the PubMed database were conducted to retrieve relevant Englishlanguage articles, and the CNKI and Wanfang database were searched for relevant Chinese-language articles. Fifteen recent guidelines or expert consensuses on the clinical nutritional management of stroke patients were published between 2013 and 2021, of which eight are from China.

Conclusions: Before providing nutritional support, swallowing, hydration, and risk of malnutrition need to be screened by a dietitian or professional. Although the initiation time of nutritional support is different in each guideline, tube feeding is preferable for patients with dysphagia. The appropriate dosage, formula, and treatment of complications need to be further studied. Also, nutritional support for stroke patients at different stages needs to be further improved. The continuous improvement and details of stroke nutrition guidelines contribute to standardized clinical nutrition practices and benefit patients.
\end{abstract}

Keywords: Stroke; enteral nutrition (EN); tube feeding (TF)

Submitted Sep 24, 2021. Accepted for publication Oct 22, 2021.

doi: 10.21037/apm-21-2922

View this article at: https://dx.doi.org/10.21037/apm-21-2922

\section{Introduction}

Over the past 30 years, stroke has been the leading cause of death in China. Although prevention and treatment strategies have improved significantly, the incidence of stroke has continually increased from 219 to 247 per 100,000 people in the period between 1989 and 2017 $(1,2)$. The mortality rate of stroke is high compared with the recent mortality rates of other diseases (3). Approximately $16.3-25 \%$ of patients who experience stroke are malnourished at admission (4,5), and as the length of hospital stay increases, this rate increases to $56.3-62 \%$ (6).
The increase in the malnutrition rate is significantly correlated with the prolongation of admission time and the decrease in functional improvement during rehabilitation (7), which affects the prognosis and mortality of the disease (8). In this study, we aimed to summarize the enteral nutrition (EN) management of stroke patients according to recent evidence.

Prof. Omorogieva Ojo and Prof. Joanne Brooke have reviewed EN for stroke patients in 2016 (9), while Chinese guidelines and expert consensuses were absence due to language restriction. To our knowledge, this is the first 
narrative review that summarizes and compares the latest EN guidelines and expert consensus for stroke patients in China with those of other countries. We summarize the latest developments in the EN guidelines and expert consensus for stroke patients in different countries, and is committed to promoting standardized enteral management of stroke patients. We present the following article in accordance with the Narrative Review reporting checklist (available at https://dx.doi.org/10.21037/apm-21-2922).

\section{Methods}

To construct this narrative review, non-systematic searches of the PubMed database were conducted to retrieve relevant English-language articles, and the CNKI and Wanfang database were searched for relevant Chinese-language articles using the following keywords: stroke, guideline, $\mathrm{EN}$, clinical nutrition, practice, and management. Articles deemed relevant to both authors, including animal studies, observational and prospective randomized clinical studies, reviews, meta-analysis, guidelines, and expert consensuses, were included.

\section{Risk factors of malnutrition in stroke patients}

The risk of malnutrition was reported as $25.8 \%$ when screening within 1.3 days after stroke in UK (10). According to different evaluation criteria the prevalence of malnutrition was $28.7 \%$ (11) to $42 \%$ (12) at admission, $76 \%$ at discharge (12) for Japanese stroke patients. Using the Mini Nutritional Assessment (MNA) (13) criteria, 5\% of Netherland stroke patients were malnourished at admission and $14 \%$ were at risk of malnutrition. Ten days after stroke, the data surged to $26 \%$ and $39 \%$ respectively (6). A multicenter prospective survey in China found that the incidence of malnutrition at first stroke patients was about $3.8 \%$ when they were admitted to the hospital, and the incidence was about $7.5 \%$ at 2 weeks after admission (14). The malnutrition rate ranges due to different evaluation criteria, population, stroke type.

It has been reported that over $90 \%$ of stroke patients are plagued by oral intake restriction, which is mainly caused by dysphagia $(15,16)$. The incidence of dysphagia ranges from $8.1 \%$ to $80 \%(17,18)$, and is the most common complication in patients with stroke and the main cause of malnutrition, especially in elderly patients $(19,20)$. A previous study showed that in patients with acute ischemic stroke (IS), $20.7 \%$ have dysphagia, and half have persistent dysphagia at hospital discharge (21). Dysphagia lasts approximately 1 month in $15 \%$ of stroke patients and may affect $11-50 \%$ at 6 months, which contributes to an increased risk of malnutrition (6,21-24). In recent years, studies have shown that malnutrition and dysphagia are risk factors for each other $(25,26)$. Patients with severe malnutrition risk were less likely to achieve full oral intake (26).

It has been reported that $30.5 \%$ of stroke patients require tube feeding (TF) (27), and 30\% need TF at discharge (28). The differences in dysphagia incidence are related to the severity of stroke (27), hematoma type, and volume (15).

The risk of malnutrition in patients without dysphagia increases if long-term energy intake insufficiency is accompanied by insufficient protein intake, suggesting that monitoring nutritional intake is necessary. The loss of consciousness $(29,30)$, poor oral hygiene (31), depression (32), reduced mobility (30), and limb and face weakness are associated with a high risk of malnutrition. During hospitalization, age, IS, National Institutes of Health Stroke Scale (NIHSS) score at admission, geriatric nutritional risk index (GNRI) at admission (33). Barthel Index feeding were associated with the nutritional status at discharge (12). Post-stroke depression often reduces a patient's appetite and impairs the recovery of the patient's ability to perform daily activities (7). Research shows that body muscle synthesis in healthy older adults is reduced by $30 \%$ and muscle strength is reduced by $16 \%$ after only 10 days of bed rest (34), which may further affect gastrointestinal motility and reattainment of full oral intake (31). In addition to dysphagia and inadequate food intake, the increased metabolic needs of patients undergoing stroke recovery also increase the risk of malnutrition. In addition, increasing age (35) and malnutrition at the time of admission (36) are also factors that increase the risk of malnutrition.

The occurrence of chronic disease and a history of medication usage at admission are significantly associated with an increased risk of malnutrition, especially in elderly patients. The presence of diabetes at admission and a history of stroke increase the risk of malnutrition by $58 \%$ and $71 \%$, respectively (4). Deficiencies in vitamins A, B, $\mathrm{C}, \mathrm{D}$ and $\mathrm{E}$ are significantly related to cerebrovascular disease, and also increase the risk of stroke and cognitive impairment in elderly patients $(5,37)$. A meta-analysis revealed that malnutrition at admission, dysphagia, previous stroke, diabetes mellitus, TF, and a reduced level of consciousness were probably correlated with an increased risk of malnutrition; however, other factors such 
as alcohol consumption, hypertension, male sex, depression, pneumonia, and infection need to be re-evaluated (29). These different conclusions suggest that additional large studies may be needed.

\section{Consequences of malnutrition}

A large number of studies have shown that malnutrition is associated with poor prognosis in patients with stroke $(14,38)$. The mortality rate increases significantly $(\mathrm{P}<0.001)$ after adjusting for age, stroke severity, and other stroke risk factors, and this correlation remains significant $(\mathrm{P}<0.001)$ (38). A multicenter cohort study in China found that after adjusting for confounding factors, the new cases of malnutrition within 2 weeks of admission (rather than malnutrition at admission) independently predicted the outcome after 3 months [odds ratio (OR) 1.37, 95\% confidence interval (CI), 1.03-1.83] (14), suggesting that new cases of malnutrition should be given more attention by medical and nursing staff. An experiment on C57BL/6 mice demonstrated that inadequate food and water intake may influence mortality following experimental stroke. After filament middle cerebral artery occlusion, appropriate nutritional support reduced the 14-day mortality by up to $75 \%$ (39).

Serum albumin and prealbumin levels can not only reflect nutritional status but also predict adverse outcomes in patients with stroke. The recurrence of stroke within 1 year is significantly increased in patients with relatively low serum albumin levels after first-ever IS (40). The serum albumin level is decreased after acute IS due to a large ischemic focus and strong catabolic reaction, and a low serum albumin level is a risk factor for worsened neurological state (41). Low serum albumin levels have been shown to be significantly associated with adverse outcomes after 3 months, with an adjusted OR (aOR) value of 1.972 (95\% CI: 1.103-4.001; $\mathrm{P}<0.001)$ (42). The recurrence and mortality rates of stroke in patients with low serum albumin are also significantly higher than those in patients with high serum albumin levels. A similar conclusion was obtained in a study on recurrence in 753 Chinese patients 1 year after stroke. In this study, patients with hypoproteinemia had a more than 3 times increase in the likelihood of suffering from recurrence $(\mathrm{aOR}=3.261,95 \% \mathrm{CI}$ : 1.914 $5.555, \mathrm{P}<0.001)$ after adjustment for other indicators (43), suggesting that low serum albumin levels may increase the risk of stroke recurrence in patients with acute IS. These studies not only confirmed the protective effect of serum albumin on the nervous system but also revealed that the nutritional status of patients was significantly related to clinical prognosis and stroke recurrence from the initial stage of IS to 1 year later. It is suggested that medical staff should pay attention not only to the nutritional level of patients at admission but also to the nutritional status of patients after discharge, as well as necessary nutrition.

Vitamin deficiency, especially the level of vitamin D (VD), which is associated with anxiety (44) and depression (45), also affects the prognoses of stroke patients. Approximately $68.8 \%$ of IS patients have VD deficiency, and only $13.6 \%$ of patients have ideal VD levels (46). The level of serum $\mathrm{VD}$ in patients with good clinical outcomes at 3 months was significantly higher than that in patients with poor outcomes. The increased level of VD in patients with acute stroke has been shown to be significantly correlated with a relatively small cerebral infarction size. Every $10 \%$ decrease in serum $25(\mathrm{OH}) \mathrm{D}$ doubles the risk of a poor outcome within 90 days (47). Previous research has shown that after adjusting for confounding factors, the functional outcomes of the four groups with the highest levels of $25(\mathrm{OH}) \mathrm{D}$ were improved at 3 months (46).

Insufficient fluid intake and fluid loss may be caused by drowsiness, dysphagia, infection-induced fever, vomiting, or diarrhea, resulting in dehydration in stroke patients (48). Approximately $62 \%$ of patients experience varying degrees of dehydration (49). Studies have suggested that dehydration adversely affects stroke patients in several ways $(50,51)$. Acute IS patients with dehydration have a higher infection rate $(\mathrm{P}=0.006)$, a worse Barthel index at discharge $(62.8 \pm 37.4$ vs. $73.4 \pm 32.4, \mathrm{P}<0.001$, adjusted $\mathrm{P}<0.001)$, a lower modified Rankin scale score $(2.7 \pm 1.6$ vs. $2.3 \pm 1.5, \mathrm{P}<0.001$, corrected $\mathrm{P}=0.009$ ), and higher hospitalization costs $(2,470.8 \pm 3,160.8$ vs. $1,901.2 \pm 2,046.8$ USD) compared to those without dehydration $(\mathrm{P}<0.001$, corrected $\mathrm{P}=0.013$ ) (52). The clinical symptoms of patients with mild to moderate dehydration (dry and wrinkled skin, decreased skin elasticity, hypotension, mucosal flushing, or dryness) are not obvious; it is also difficult to diagnose clinical symptoms in elderly patients with dehydration (53). Since there is no current common diagnostic standard, it is difficult to diagnose dehydration accurately (54).

\section{Nutritional management}

We have identified 15 guidelines and expert consensus statements, which are discussed and summarized as follows (55-69). Since the Veterans Affairs (VA)/Departments 
of Defense (DOD) guidelines (65) only have one recommendation about $\mathrm{TF}$, guideline for primary care of IS [2021] (69) just mention the assessment of swallowing, patients with dysphagia receive nutrition support within 7 days, nasogastric tube (NGT) for patients with short-term dysphagia, and gastrostomy tube for long-term, these two are not listed in Table 1.

\section{When, by whom, and bow to judge whether a patient needs nutritional support}

Identifying patients with nutritional risk or malnourishment is a prerequisite for nutritional management. Ten out of the 15 guidelines recommend that stroke patients should be screened for nutritional risk, and 5 of them suggested as soon as possible after admission $(55,56,59,62,63), 2$ of them proposed within $24 \mathrm{~h}(66,67), 3$ of them indicated within $48 \mathrm{~h}(57,58,64)$. In addition, 3 of them required rescreen at least weekly thereafter $(55,56,62)$, and regularly during rehabilitation (64). A structured tool has been recommended for screening malnutrition $(55,56,59,64,66)$. Body mass index (BMI) and percentage of unintentional weight loss should be assessed during screening (62). The malnutrition universal screening tool (MUST) (70) is a recommended and established nutritional screening tool $(58,62)$ that can be used on stroke patients to identify those at risk of malnutrition (38). Nutrition Risk Screening 2002 (NRS2002) (71) is recommended by three Chinese guidelines $(60,63,68)$. Mini nutritional assessment-short form (MNA-SF) was recommended as the preferred tool for geriatrics by nursing practice guideline for $\mathrm{EN}$ in patients with stroke (68) due to its relatively high prediction accuracy (reliability, validity, sensitivity, and specificity). However, Chinese dysphagia and nutrition management manual for acute stroke patients (66) does not recommend it as the preferred screening tool as it has no good correlation and poor specificity with the mortality, complications and other outcome indicators of the elderly. A study from China showed that both NRS2002 and MUST can be used to screen for malnutrition risk in patients with stroke (30). Screening for malnutrition and the risk of malnutrition should be carried out by trained staff $(56,62)$ or by first visited nurse (66). In addition to nutritional risk, screening for swallowing (55,57,58,60,63,67-69) and hydration $(56,60)$ status on admission is also necessary $(59,62,66)$. The guidelines suggest that routine nutritional supplementation for patients without oral intake restriction, malnutrition, or risk of malnutrition is unnecessary $(55,56,58,62,67)$. Abnormal results of swallowing and nutrition screening should be further assessed, and conditions should be managed (55,56,59,62,64,66-69). An individualized nutrition care plan that is developed and monitored by professionals is recommended $(56,58,62,64,67)$.

\section{When, by whom, and how to deliver EN}

$\mathrm{EN}$ is the most natural way in which nutrients are absorbed (72); the theory of "if the gut works, use it" is widely recognized. EN can be administered via a nasogastric (NG), nasoduodenal or nasojejunal (NJ) TF; percutaneous endoscopic gastrostomy (PEG); or percutaneous endoscopic jejunostomy (PEJ) (72), which is the first choice for patients who can tolerate EN (63). EN can be accomplished by a continuous, set-rate, or intermittent method (72). Figure 1 displays a flow chart showing how to choose the delivery methods of EN for stroke patients after admission.

$\mathrm{EN}$ is recommended to be administered within $24 \mathrm{~h}$ $(56,62,66)$ or within $24-48 \mathrm{~h}(63,67)$ or not more than $72 \mathrm{~h}(58)$, or within 7 days $(61-63,66,69)$ for stroke patients who are unable to maintain adequate nutrition, are already malnourished, or have eating difficulties. A NGT should be considered within $72 \mathrm{~h}$ (58) or within the first 7 days (61) for patients who cannot consume adequate nutrition or water orally, or have prolonged severe dysphagia lasting for more than 7 days (58). NGT is the first choice for patients who receive EN within 2 weeks (63). A nasal bridle tube can be applied if the NGT is intolerable or rejected, EN is expected to last for more than 14 days, or the NGT needs frequent replacement (56). Gastrostomy feeding (PEG or PEJ) can be considered if the NGT cannot be tolerated $(56,62)$, if the patient is unable to consume adequate food or fluids orally over $2-3$ weeks $(67)$ or 4 weeks $(56,57,67)$ after the onset of stroke or if the patient has a high risk of long-term malnutrition (56). PEG should be considered within 1 week if mechanical ventilation lasts for longer than $48 \mathrm{~h} \mathrm{(58)}$ or after $14-28$ days if EN is needed for a long period of time ( $>4$ weeks) $(55,57,58,61,64,68)$. If conditions permit, PEG is recommended for patients requiring long-term $\mathrm{EN}$ (63). A gastrostomy tube is a more sustainable choice than NGT for patients with dysphagia in the post-acute phase of stroke (65). For patients with gastroesophageal reflux and aspiration, the NJ tube (NJT) may be the best choice (57) and continuous drip feeding was recommended $(67,68)$. 


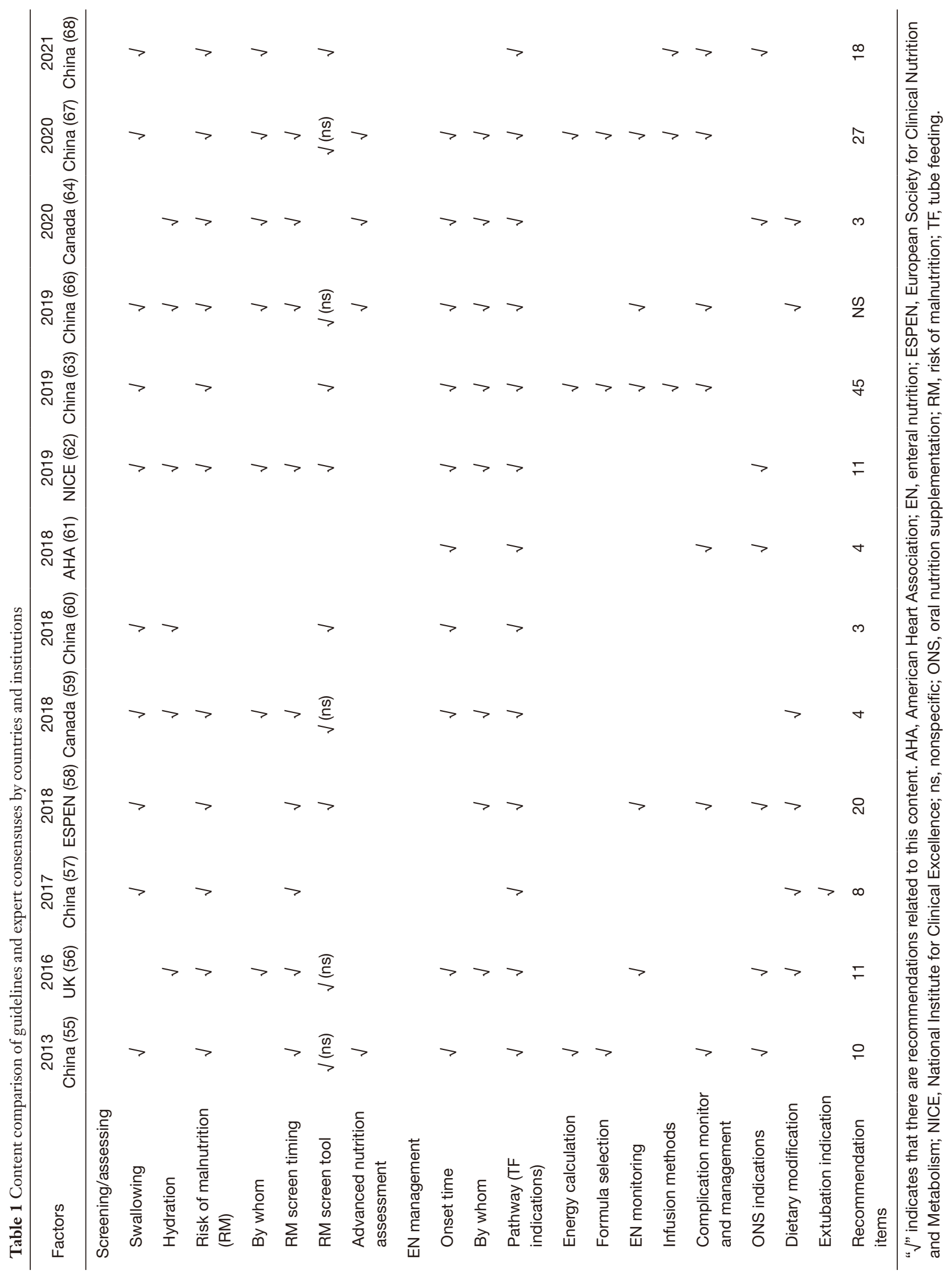




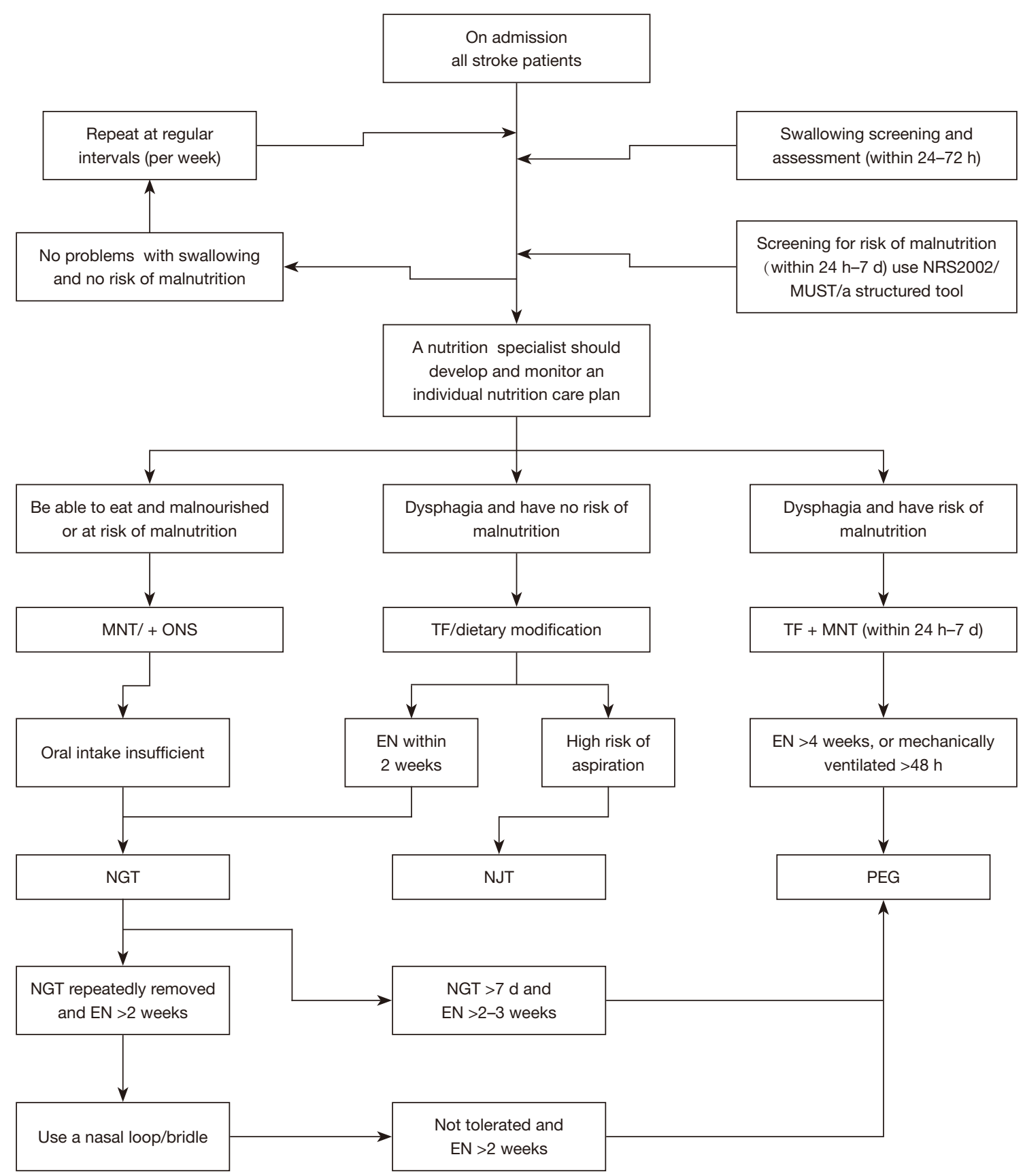

Figure 1 Tube feeding selection flowchart. NRS2002, Nutritional Risk Screening 2002; MUST, Malnutrition Universal Screening Tool; MNT, medical nutrition therapy; ONS, oral nutritional supplements; TF, tube feeding; NGT, nasogastric tube; EN, enteral nutrition; NJT, nasoduodenal or nasojejunal tube; PEG, percutaneous endoscopic gastrostomy.

Both the amount and rate of EN administered should begin low and be gradually increased, from $20-50 \mathrm{~mL} / \mathrm{h}$ on the first day to $80-100 \mathrm{~mL} / \mathrm{h}$ on the next day, and the infusion should be completed in 12-24 h and reach the full amount within 3 days $(63,67)$. The amount of nasal feeding should not exceed $200 \mathrm{~mL}$ each time, and the interval should be established according to the total amount of the whole day and the digestion and absorption of the 
patient (66). Also, a dietitian or nutritional specialist should perform a nutritional assessment, provide advice, and conduct monitoring if a patient is at risk of malnutrition $(56,58,59,62,63,67,68)$, TF placement, dietary modification $(56,57,59,64,66)$, or requires thickened liquids $(56,58,66)$.

According to previous reports, looped NGT feeding is associated with a $17 \%$ higher volume of feed and fluids, as well as fewer NGT reinsertions and electrolyte abnormalities compared to conventional adhesive dressings (73). However, there were no differences in case fatality, composite death, or dependency rates between NGT and PEG, but there were fewer treatment failures, less gastrointestinal bleeding, higher feed delivery, and higher albumin concentrations in the PEG group than in the NGT group (8). Another study demonstrated that there was no difference in the outcome of nutritional status, including weight change from baseline and mid-arm circumference at the endpoint, between the PEG and NGT groups; additionally, there were no differences in mortality, overall adverse events at any follow-up time point, and pneumonia (aspiration) rates (74).

For stroke patients who are able to eat but are malnourished (55) or those at risk of malnutrition $(58,61)$, oral nutrition supplements are recommended (68). Fluid balance and nutritional intake for patients who receive texture-modified diets or thickened fluids should be monitored, as they may lead to reduced energy and fluid intake (58).

\section{How much and what to deliver}

Indirect calorimetry to estimate energy consumption is recommended $(55,67)$. If indirect calorimetry is not available, the Schofield equation can be used to calculate the basal metabolic rate, or empirical formulas can be used to calculate the energy demand of patients and adjust the basic substrate supply according to the severity of the disease (55).

Energy requirements should be estimated as $25-$ $35 \mathrm{kcal} / \mathrm{kg}$ body weight for moderately ambulatory patients [Glasgow coma scale (GCS) score $>12$ or Acute Physiology and Chronic Health Evaluation (APACHE II) score $\leq 16$; $20-25 \mathrm{kcal} / \mathrm{kg}$ body weight for bedridden patients, with a sugar-lipid mass ratio of 7:3-6:4 and calorie nitrogen ratio of 100-150:1; and 20-25 kcal/ $\mathrm{kg}$ body weight for severe acute stress patients, with a sugar-lipid mass ratio of 5:5 and a calorie nitrogen ratio of 100:1 $(63,67)$. Dietary fiber intake should be reach $25-30 \mathrm{~g} /$ day $(55)$. It is recommended to estimate the daily protein demand of patients through the determination of 24-h urinary urea nitrogen (UUN) (67).

\section{Complications and management}

Diarrhea refers to thin stools $>3$ times/day or $>200 \mathrm{~g} /$ day. The incidence of diarrhea ranges from $29.4 \%$ to $38 \%$ $(75,76)$ and often results in electrolyte imbalance, dehydration, perianal skin breakdown, and wound contamination (77). If unable to control diarrhea, clinicians often stop EN, resulting in inadequate nutrition intake (78). Factors associated with the occurrence of diarrhea include the following: EN formulation, supply mode, and speed; EN initiation time and duration $(79,80)$; EN contamination; medication use (antibiotics, proton-pump inhibitors, prokinetics, laxatives); infectious etiologies (77); as well as the NIHSS score $(81,82)$, GCS score $(81,83)$, length of stay $(80,81)$, antibiotic use $(80,81)$ and hypoalbuminemia. A 24-h delivery set hang time is associated with a lower risk of diarrhea and longer diarrhea-free survival in patients with acute stroke under TF than a 72/96-h delivery set hang time (84). Furthermore, 7 days or more of enteral TF is independently associated with diarrhea (82). Altering the composition of polymeric enteral diets to include a rapidly fermentable fiber source can prevent the onset of enteral feeding-related diarrhea (85).

A recent hypothesis suggests that diarrhea may be induced by the presence of poorly absorbed short-chain carbohydrates, including fermentable oligosaccharides (FOs), disaccharides (Ds), monosaccharides (Ms), and polyols (FODMAP) (86). Individuals with digestive impairment and problems with the absorption of these carbohydrates frequently exhibit intolerance to foods rich in FODMAPs, presenting abdominal distension, colic, and diarrhea, among other intestinal symptoms (87). A previous study pointed out that FODMAPs at levels in current enteral formulas are not involved in the pathophysiology of enteral feeding-related diarrhea, and they are likely to be beneficial rather than detrimental (88).

The incidence of diarrhea can be reduced by slowing the infusion speed, reducing the total infusion amount, administering an isotonic nutritional formula, strictly abiding by aseptic techniques, paying attention to the diagnosis of antimicrobial-associated diarrhea (63), correcting hypoproteinemia according to the clinical situation, avoiding the long-term use of broad-spectrum antibiotics, maintaining proper temperatures, administering lactose-free formulas to lactose-intolerant patients (55), administering continuous infusion, and including fiber in enteral diets (89).

Stroke associated pneumonia (SAP) is one of the most fatal complications of stroke (90), and a high NIHSS 
score (91), dysphagia (91), and proton-pump inhibitor use (92) were found to be correlated with an increased risk of aspiration pneumonia. Disturbance of consciousness after stroke, aspiration caused by swallowing dysfunction and immunosuppression caused by stroke are considered to be the main pathogenesis of SAP (93). Whether NGT placement increases the risk of pneumonia is not clear $(94,95)$. Prophylactic antibiotics were reported to have no effect on pneumonia in stroke patients (96) and is not recommended (97) Previous studies have shown that a three-step multidisciplinary dysphagia screening intervention (95), a formal screening protocol and early dysphagia screening (98) and training for swallowing (97), a structured protocol incorporating cough reflex testing (99), an integrated team approach and dysphagia clinical pathway, and implementing oral hygiene protocols $(61,97)$ had positive impacts on incidence of SAP $(100,101)$. Raising the patient's bedside $30^{\circ}-45^{\circ}$ is an effective measure to prevent SAP $(68,97)$. Compared with intermittent feeding, continuous feeding helps reduce lung infection and aspiration (68). For patients with pyloric obstruction, gastroparesis, esophageal reflux, or aspiration, the use of post-pyloric TF can reduce the incidence of SAP (97).

Vomiting and abdominal distension can be managed by slowing the speed of infusion, reducing the total infusion amount, identifying causes, treating symptoms, and changing to parenteral nutrition when symptoms are not relieved (63).

\section{Conclusions}

The consistency and effectiveness of different nutritional screening tools in stroke patients requires further investigation in large-scale studies in Chinese populations. A simple, fast, easy to understand, and accurate nutritional risk screening tool is helpful in identifying patients who will benefit from nutrition management. Due to the low acceptance of PEG by Chinese patients, there is a lack of research on nutrition satisfaction, prognoses, and complications associated with different feeding methods (NGT, NJT, and PEG) in patients who require long-term ( $>4$ weeks) EN, especially those with a long-term indwelling NGT that should be replaced by PEG but is not.

Nutritional management is an important part of the treatment of stroke patients. Standard nutritional management contributes to the treatment and rehabilitation of stroke. In China, most hospitals do not have nutrition departments or dietitians, and the nutritional management of stroke patients is supervised and implemented by clinicians. Therefore, continuous improvements in nutritional management recommended by the stroke guidelines will be helpful for clinicians and dietitians to standardize the nutritional management of stroke patients.

\section{Acknowledgments}

Prof. Yulin Gao merits our gratitude for his advice in revising the review.

Funding: None.

\section{Footnote}

Reporting Checklist: The authors have completed the Narrative Review reporting checklist. Available at https:// dx.doi.org/10.21037/apm-21-2922

Conflicts of Interest: All authors have completed the ICMJE uniform disclosure form (available at https://dx.doi. org/10.21037/apm-21-2922). The authors have no conflicts of interest to declare.

Ethical Statement: The authors are accountable for all aspects of the work in ensuring that questions related to the accuracy or integrity of any part of the work are appropriately investigated and resolved.

Open Access Statement: This is an Open Access article distributed in accordance with the Creative Commons Attribution-NonCommercial-NoDerivs 4.0 International License (CC BY-NC-ND 4.0), which permits the noncommercial replication and distribution of the article with the strict proviso that no changes or edits are made and the original work is properly cited (including links to both the formal publication through the relevant DOI and the license). See: https://creativecommons.org/licenses/by-nc-nd/4.0/.

\section{References}

1. Shi FL, Hart RG, Sherman DG, et al. Stroke in the People's Republic of China. Stroke 1989;20:1581-5.

2. Wang W, Jiang B, Sun H, et al. Prevalence, Incidence, and Mortality of Stroke in China: Results from a Nationwide Population-Based Survey of 480687 Adults. Circulation 2017;135:759-71.

3. Thrift AG, Thayabaranathan T, Howard G, et al. Global stroke statistics. Int J Stroke 2017;12:13-32. 
4. Corrigan ML, Escuro AA, Celestin J, et al. Nutrition in the stroke patient. Nutr Clin Pract 2011;26:242-52.

5. Sánchez-Moreno C, Jiménez-Escrig A, Martín A. Stroke: roles of B vitamins, homocysteine and antioxidants. Nutr Res Rev 2009;22:49-67.

6. Mosselman MJ, Kruitwagen CL, Schuurmans MJ, et al. Malnutrition and risk of malnutrition in patients with stroke: prevalence during hospital stay. J Neurosci Nurs 2013;45:194-204.

7. Mould J. Nurses 'must' control of the nutritional needs of stroke patients. Br J Nurs 2009;18:1410-4.

8. Geeganage C, Beavan J, Ellender S, et al. Interventions for dysphagia and nutritional support in acute and subacute stroke. Cochrane Database Syst Rev 2012;10:CD000323.

9. Ojo O, Brooke J. The Use of Enteral Nutrition in the Management of Stroke. Nutrients 2016;8:827.

10. Fluck D, Fry CH, Gulli G, et al. Association of risk of malnutrition with adverse outcomes and early support on discharge in acute stroke patients without prestroke disability: A multicenter, registry-based cohort study. Nutr Clin Pract 2021. [Epub ahead of print].

11. Nozoe M, Yamamoto M, Masuya R, et al. Prevalence of Malnutrition Diagnosed with GLIM Criteria and Association with Activities of Daily Living in Patients with Acute Stroke. J Stroke Cerebrovasc Dis 2021;30:105989.

12. Sato M, Ido Y, Yoshimura Y, et al. Relationship of Malnutrition During Hospitalization With Functional Recovery and Postdischarge Destination in Elderly Stroke Patients. J Stroke Cerebrovasc Dis 2019;28:1866-72.

13. Vellas B, Guigoz Y, Garry PJ, et al. The Mini Nutritional Assessment (MNA) and its use in grading the nutritional state of elderly patients. Nutrition 1999;15:116-22.

14. Zhang J, Zhao X, Wang A, et al. Emerging malnutrition during hospitalisation independently predicts poor 3-month outcomes after acute stroke: data from a Chinese cohort. Asia Pac J Clin Nutr 2015;24:379-86.

15. Maeshima S, Osawa A, Yamane F, et al. Dysphagia following acute thalamic haemorrhage: clinical correlates and outcomes. Eur Neurol 2014;71:165-72.

16. Buoite Stella A, Gaio M, Furlanis G, et al. Fluid and energy intake in stroke patients during acute hospitalization in a stroke unit. J Clin Neurosci 2019;62:27-32.

17. Takizawa C, Gemmell E, Kenworthy J, et al. A Systematic Review of the Prevalence of Oropharyngeal Dysphagia in Stroke, Parkinson's Disease, Alzheimer's Disease, Head Injury, and Pneumonia. Dysphagia 2016;31:434-41.

18. Xia W, Zheng C, Zhu S, et al. Does the addition of specific acupuncture to standard swallowing training improve outcomes in patients with dysphagia after stroke? a randomized controlled trial. Clin Rehabil 2016;30:237-46.

19. Sura L, Madhavan A, Carnaby G, et al. Dysphagia in the elderly: management and nutritional considerations. Clin Interv Aging 2012;7:287-98.

20. Drozdz D, Mancopes R, Silva AM, et al. Analysis of the level of Dysphagia, anxiety, and nutritional status before and after speech therapy in patients with stroke. Int Arch Otorhinolaryngol 2014;18:172-7.

21. Norman K, Pichard C, Lochs H, et al. Prognostic impact of disease-related malnutrition. Clin Nutr 2008;27:5-15.

22. Ickenstein GW, Höhlig C, Prosiegel M, et al. Prediction of outcome in neurogenic oropharyngeal dysphagia within 72 hours of acute stroke. J Stroke Cerebrovasc Dis 2012;21:569-76.

23. Mann G, Hankey GJ, Cameron D. Swallowing disorders following acute stroke: prevalence and diagnostic accuracy. Cerebrovasc Dis 2000;10:380-6.

24. Smithard DG, O'Neill PA, Parks C, et al. Complications and outcome after acute stroke. Does dysphagia matter? Stroke 1996;27:1200-4.

25. Carrión S, Cabré M, Monteis R, et al. Oropharyngeal dysphagia is a prevalent risk factor for malnutrition in a cohort of older patients admitted with an acute disease to a general hospital. Clin Nutr 2015;34:436-42.

26. Nishioka S, Okamoto T, Takayama M, et al. Malnutrition risk predicts recovery of full oral intake among older adult stroke patients undergoing enteral nutrition: Secondary analysis of a multicentre survey (the APPLE study). Clin Nutr 2017;36:1089-96.

27. Arnold M, Liesirova K, Broeg-Morvay A, et al. Dysphagia in Acute Stroke: Incidence, Burden and Impact on Clinical Outcome. PLoS One 2016;11:e0148424.

28. Wilmskoetter J, Bonilha L, Martin-Harris B, et al. Factors Influencing Oral Intake Improvement and Feeding Tube Dependency in Patients with Poststroke Dysphagia. J Stroke Cerebrovasc Dis 2019;28:1421-30.

29. Chen N, Li Y, Fang J, et al. Risk factors for malnutrition in stroke patients: A meta-analysis. Clin Nutr 2019;38:127-35.

30. Wang J, Luo B, Xie Y, et al. Evaluation methods on the nutritional status of stroke patients. Eur Rev Med Pharmacol Sci 2014;18:3902-7.

31. Nishioka S, Yamasaki K, Ogawa K, et al. Impact of nutritional status, muscle mass and oral status on recovery of full oral intake among stroke patients receiving enteral nutrition: A retrospective cohort study. Nutr Diet 2020;77:456-66. 
32. Kim Y, Kim MC, Park HS, et al. Association of the Anxiety/Depression with Nutrition Intake in Stroke Patients. Clin Nutr Res 2018;7:11-20.

33. Bouillanne O, Morineau G, Dupont C, et al. Geriatric Nutritional Risk Index: a new index for evaluating at-risk elderly medical patients. Am J Clin Nutr 2005;82:777-83.

34. Kortebein P, Ferrando A, Lombeida J, et al. Effect of 10 days of bed rest on skeletal muscle in healthy older adults. JAMA 2007;297:1772-4.

35. Hsieh DY, Hung JW, Chang KC, et al. Malnutrition in Acute Stroke Patients Stratified by Stroke SeverityA Hospital Based Study. Acta Neurol Taiwan 2017;26(3):120-7.

36. Deng $\mathrm{X}$, Huang L, Jiang H, et al. Risk factors analysis of undernutrition, complications and poor prognosis in acute stroke patients. J Clin Neurol 2013;26:31-4.

37. Aquilani R, Sessarego P, Iadarola P, et al. Nutrition for brain recovery after ischemic stroke: an added value to rehabilitation. Nutr Clin Pract 2011;26:339-45.

38. Gomes F, Emery PW, Weekes CE. Risk of Malnutrition Is an Independent Predictor of Mortality, Length of Hospital Stay, and Hospitalization Costs in Stroke Patients. J Stroke Cerebrovasc Dis 2016;25:799-806.

39. Lourbopoulos A, Mamrak U, Roth S, et al. Inadequate food and water intake determine mortality following stroke in mice. J Cereb Blood Flow Metab 2017;37:2084-97.

40. He H, Guo J. Serum albumin: a risk of stroke? Am J Emerg Med 2017;35:186-7.

41. Bielewicz J, Kurzepa J, Czekajska-Chehab E, et al. Worse Neurological State During Acute Ischemic Stroke is Associated with a Decrease in Serum Albumin Levels. J Mol Neurosci 2016;58:493-6.

42. Babu MS, Kaul S, Dadheech S, et al. Serum albumin levels in ischemic stroke and its subtypes: correlation with clinical outcome. Nutrition 2013;29:872-5.

43. Zhang Q, Lei YX, Wang Q, et al. Serum albumin level is associated with the recurrence of acute ischemic stroke. Am J Emerg Med 2016;34:1812-6.

44. Wu C, Ren W, Cheng J, et al. Association Between Serum Levels of Vitamin D and the Risk of Post-Stroke Anxiety. Medicine (Baltimore) 2016;95:e3566.

45. Han B, Lyu Y, Sun H, et al. Low serum levels of vitamin $\mathrm{D}$ are associated with post-stroke depression. Eur J Neurol 2015;22:1269-74.

46. Park KY, Chung PW, Kim YB, et al. Serum Vitamin D Status as a Predictor of Prognosis in Patients with Acute Ischemic Stroke. Cerebrovasc Dis 2015;40:73-80.

47. Turetsky A, Goddeau RP Jr, Henninger N. Low Serum
Vitamin D Is Independently Associated with Larger Lesion Volumes after Ischemic Stroke. J Stroke Cerebrovasc Dis 2015;24:1555-63.

48. Rowat A. Malnutrition and dehydration after stroke. Nurs Stand 2011;26:42-6.

49. Rowat A, Graham C, Dennis M. Dehydration in hospitaladmitted stroke patients: detection, frequency, and association. Stroke 2012;43:857-9.

50. Acciarresi M, Bogousslavsky J, Paciaroni M. Poststroke fatigue: epidemiology, clinical characteristics and treatment. Eur Neurol 2014;72:255-61.

51. Buoite Stella A, Gaio M, Furlanis G, et al. Prevalence of hypohydration and its association with stroke severity and independence outcomes in acute ischemic stroke patients. J Clin Neurosci 2020;72:281-6.

52. Liu CH, Lin SC, Lin JR, et al. Dehydration is an independent predictor of discharge outcome and admission cost in acute ischaemic stroke. Eur J Neurol 2014;21:1184-91.

53. Vivanti A, Harvey K, Ash S, et al. Clinical assessment of dehydration in older people admitted to hospital: what are the strongest indicators? Arch Gerontol Geriatr 2008;47:340-55.

54. Bahouth MN, Gottesman RF, Szanton SL. Primary 'dehydration' and acute stroke: a systematic research review. J Neurol 2018;265:2167-81.

55. Wang Y, Zhao X, Wang S. Chinese experts' consensus on dysphagia and Nutrition Management in Stroke patients. Chinese Journal of Stroke 2013;8:973-83.

56. Bowen A, James M, Young G, editors. 2016 National clinical guideline for stroke. Royal College of Physicians, 2016.

57. Zhao T, Zhao J. Guidelines for early rehabilitation of stroke in China. Chinese Journal of Neurology 2017;50:405-12.

58. Burgos R, Bretón I, Cereda E, et al. ESPEN guideline clinical nutrition in neurology. Clin Nutr 2018;37:354-96.

59. Boulanger JM, Lindsay MP, Gubitz G, et al. Canadian Stroke Best Practice Recommendations for Acute Stroke Management: Prehospital, Emergency Department, and Acute Inpatient Stroke Care, 6th Edition, Update 2018. Int J Stroke 2018;13:949-84.

60. Peng B, Wu B. Chinese guidelines for diagnosis and treatment of acute ischemic stroke 2018. Chinese Journal of Neurology 2018;51:666-82.

61. Powers WJ, Rabinstein AA, Ackerson T, et al. 2018 Guidelines for the Early Management of Patients With Acute Ischemic Stroke: A Guideline for Healthcare 
Professionals From the American Heart Association/ American Stroke Association. Stroke 2018;49:e46-e110.

62. National Institute for Health and Clinical Excellence. National Institute for Health and Care Excellence: Clinical Guidelines. Stroke and transient ischaemic attack in over 16s: diagnosis and initial management. London: National Institute for Health and Care Excellence (UK), 2019.

63. Su Y, Pan S, Peng B, et al. Chinese expert consensus on enteral nutrition support for neurological disease (the second version). Chinese Journal of Clinical Nutrition 2019;27:193-203.

64. Teasell R, Salbach NM, Foley N, et al. Canadian Stroke Best Practice Recommendations: Rehabilitation, Recovery, and Community Participation following Stroke. Part One: Rehabilitation and Recovery Following Stroke; 6th Edition Update 2019. Int J Stroke 2020;15:763-88.

65. Management of Stroke Rehabilitation [database on the Internet] 2019. Available online: https:// www.healthquality.va.gov/guidelines/Rehab/stroke/ VADoDStrokeRehabCPGFinal8292019.pdf

66. Wang Y, Wang S, Zhao X, et al. et al. Chinese Dysphagia and Nutrition Management Manual for Acute stroke Patients. Chinese Journal of Stroke 2019;14:1153-69.

67. Wang Y, Zhao X, Wang S, et al. Chinese Consensus on Standardized Nutrition Management in Patients with Stroke. Chinese Journal of Stroke 2020;6:681-9.

68. Chinese Evidence-based Medicine Center, West China Hospital, Sichuan University; Nursing Management Professional Committee, Chinese Nursing Association; Chinese Neurosurgical Society, Chinese Medical Association. Nursing practice guideline for enteral nutrition in patients with stroke. Chinese Journal of Evidence-Based Medicine 2021;21:628-41.

69. Chinese Medical Association, Journal of Chinese Medical Association, General Practice Branch of Chinese Medical Association, et al. Guideline for primary care of ischemic stroke (2021). Chinese Journal of General Practitioners 2021;20:927- 46.

70. Elia M. The 'MUST'report. Nutritional screening for adults: a multidisciplinary responsibility. Development and use of the 'malnutrition universal screening tool'(MUST) for adults. The British Association for Parenteral and Enteral Nutrition, 2003.

71. Kondrup J, Allison SP, Elia M, et al. ESPEN guidelines for nutrition screening 2002. Clin Nutr 2003;22:415-21.

72. Baiu I, Spain DA. Enteral Nutrition. JAMA 2019;321:2040.

73. Beavan J, Conroy SP, Harwood R, et al. Does looped nasogastric tube feeding improve nutritional delivery for patients with dysphagia after acute stroke? A randomised controlled trial. Age Ageing 2010;39:624-30.

74. Gomes CA Jr, Andriolo RB, Bennett C, et al. Percutaneous endoscopic gastrostomy versus nasogastric tube feeding for adults with swallowing disturbances. Cochrane Database Syst Rev 2015;(5):CD008096.

75. Schmidt SB, Kulig W, Winter R, et al. The effect of a natural food based tube feeding in minimizing diarrhea in critically ill neurological patients. Clin Nutr 2019;38:332-40.

76. Rahman KM, Khan SU, Hasan AH, et al. Nasogastric Tube Feeding Practice and Associated Complication in stroke Patients in a Tertiary Care Hospital in Bangladesh. Journal of National Institute of Neurosciences Bangladesh 2018;4:23-7.

77. Chang SJ, Huang HH. Diarrhea in enterally fed patients: blame the diet? Curr Opin Clin Nutr Metab Care 2013;16:588-94.

78. McClave SA, Taylor BE, Martindale RG, et al. Guidelines for the Provision and Assessment of Nutrition Support Therapy in the Adult Critically Ill Patient: Society of Critical Care Medicine (SCCM) and American Society for Parenteral and Enteral Nutrition (A.S.P.E.N.). JPEN J Parenter Enteral Nutr 2016;40:159-211.

79. Chen W, Chen J, Lin B. An overview of diarrhea in ICU patients receiving enteral nutrition. Int J Clin Exp Med 2019;12:11893-7.

80. Halmos EP, Muir JG, Barrett JS, et al. Diarrhoea during enteral nutrition is predicted by the poorly absorbed shortchain carbohydrate (FODMAP) content of the formula. Aliment Pharmacol Ther 2010;32:925-33.

81. Xiang Y, Li F, Peng J, et al. Risk Factors and Predictive Model of Diarrhea Among Patients with Severe Stroke. World Neurosurg 2020;136:213-9.

82. Arevalo-Manso JJ, Martinez-Sanchez P, Juarez-Martin B, et al. Enteral tube feeding of patients with acute stroke: when does the risk of diarrhoea increase? Intern Med J 2014;44:1199-204.

83. Fu X, Wong KS, Wei JW, et al. Factors associated with severity on admission and in-hospital mortality after primary intracerebral hemorrhage in China. Int J Stroke 2013;8:73-9.

84. Arevalo-Manso JJ, Martinez-Sanchez P, Juarez-Martin $\mathrm{B}$, et al. Preventing diarrhoea in enteral nutrition: the impact of the delivery set hang time. Int J Clin Pract 2015;69:900-8.

85. Silk DBA, Bowling T. Pathophysiology of Enteral Feeding Diarrhea: The Intestinal Responses to Enteral Feeding 
Rather Than Any Role of FODMAPs. JPEN J Parenter Enteral Nutr 2017;41:1259-61.

86. Mezzomo TR, Sampaio IR, Fiori LS, et al. Content of Poorly Absorbed Short-Chain Carbohydrates (FODMAP) in Enteral Homemade Diets. Nutr Clin Pract 2019;34:264-71.

87. Pedersen N, Ankersen DV, Felding M, et al. LowFODMAP diet reduces irritable bowel symptoms in patients with inflammatory bowel disease. World J Gastroenterol 2017;23:3356-66.

88. Halmos EP, Bogatyrev A, Ly E, et al. Challenges of Quantifying FODMAPs in Enteral Nutrition Formulas: Evaluation of Artifacts and Solutions. JPEN J Parenter Enteral Nutr 2017;41:1262-71.

89. Elia M, Engfer MB, Green CJ, et al. Systematic review and meta-analysis: the clinical and physiological effects of fibre-containing enteral formulae. Aliment Pharmacol Ther 2008;27:120-45.

90. Li J, Zhang P, Wu S, et al. Stroke-related complications in large hemisphere infarction: incidence and influence on unfavorable outcome. Ther Adv Neurol Disord 2019;12:1756286419873264.

91. Xu Z, Gu Y, Li J, et al. Dysphagia and aspiration pneumonia in elderly hospitalization stroke patients: Risk factors, cerebral infarction area comparison. J Back Musculoskelet Rehabil 2019;32:85-91.

92. Arai N, Nakamizo T, Ihara H, et al. Histamine H2Blocker and Proton Pump Inhibitor Use and the Risk of Pneumonia in Acute Stroke: A Retrospective Analysis on Susceptible Patients. PLoS One 2017;12:e0169300.

93. Hannawi Y, Hannawi B, Rao CP, et al. Stroke-associated pneumonia: major advances and obstacles. Cerebrovasc

Cite this article as: Gong L, Wang Y, Shi J. Enteral nutrition management in stroke patients: a narrative review. Ann Palliat Med 2021;10(10):11191-11202. doi: 10.21037/apm-21-2922
Dis 2013;35:430-43.

94. Schwarz M, Coccetti A, Murdoch A, et al. The impact of aspiration pneumonia and nasogastric feeding on clinical outcomes in stroke patients: A retrospective cohort study. J Clin Nurs 2018;27:e235-41.

95. Kalra L, Hodsoll J, Irshad S, et al. Association between nasogastric tubes, pneumonia, and clinical outcomes in acute stroke patients. Neurology 2016;87:1352-9.

96. Vermeij JD, Westendorp WF, Dippel DW, et al. Antibiotic therapy for preventing infections in people with acute stroke. Cochrane Database Syst Rev 2018;1:CD008530.

97. Wang YJ, Chen YG, Lv CZ, et al. An updated Chinese consensus statement on stroke-associated pneumonia 2019. Asian Pacific Journal of Tropical Medicine 2019;12:1-11.

98. Eltringham SA, Kilner K, Gee M, et al. Impact of Dysphagia Assessment and Management on Risk of Stroke-Associated Pneumonia: A Systematic Review. Cerebrovasc Dis 2018;46:99-107.

99. Perry SE, Miles A, Fink JN, et al. The Dysphagia in Stroke Protocol Reduces Aspiration Pneumonia in Patients with Dysphagia Following Acute Stroke: a Clinical Audit. Transl Stroke Res 2019;10:36-43.

100.Palli C, Fandler S, Doppelhofer K, et al. Early Dysphagia Screening by Trained Nurses Reduces Pneumonia Rate in Stroke Patients: A Clinical Intervention Study. Stroke 2017;48:2583-5.

101.Aoki S, Hosomi N, Hirayama J, et al. The Multidisciplinary Swallowing Team Approach Decreases Pneumonia Onset in Acute Stroke Patients. PLoS One 2016;11:e0154608.

(English Language Editor: A. Kassem) 